\title{
Gesetzmäßigkeiten in der Unternehmensführung? Unternehmensführung als das Verfügen über Notwendigkeiten
}

Aaken, Dominik ; Kirsch, Werner ; Seidl, David

\begin{abstract}
Taking the debate on evidence-based management and success-factor research as a point of departure, this paper discusses three different views of the role of identified regularities for management practice: (1) acceptance of regularities, where regularities are understood as delimiting the scope of action, (2) use of regularities, where regularities are understood as restrictions that change over time and (3) management of regularities, where regularities are understood as temporary invariances that can be managed by the firm. Reflecting on the scientific status of regularities in the social sciences, we argue that the first two views are based on a limited understanding of the nature of management sciences.
\end{abstract}

DOI: https://doi.org/10.5771/0042-059X-2015-1-54

Posted at the Zurich Open Repository and Archive, University of Zurich ZORA URL: https://doi.org/10.5167/uzh-173249

Journal Article

Published Version

Originally published at:

Aaken, Dominik; Kirsch, Werner; Seidl, David (2015). Gesetzmäßigkeiten in der Unternehmensführung? Unternehmensführung als das Verfügen über Notwendigkeiten. Die Unternehmung, 69(1):54-66.

DOI: https://doi.org/10.5771/0042-059X-2015-1-54 


\title{
Gesetzmäßigkeiten in der Unternehmensführung?
}

\author{
Unternehmensführung als das Verfügen über Notwendigkeiten
}
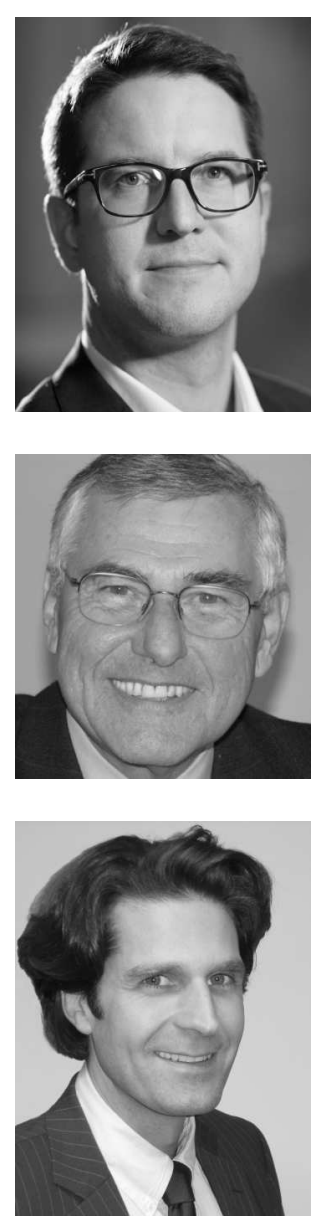

\section{Dominik van Aaken, Werner Kirsch und David Seidl}

Erfolgsfaktorenforschung, Evidence-Based Management, Management, Strategisches Management, Unternehmensführung, Wissenschaftstheorie

Evidence-based management, management, philosophy of science, strategic management, success-factor research

Bezugnehmend auf die Diskussion zum Evidence-Based Management und zur Erfolgsfaktorenforschung legen wir in diesem Beitrag drei unterschiedliche Auffassungen zum Umgang mit (vermeintlichen) Gesetzmäßigkeiten in der Unternehmensführung dar: (1) Führung als „Akzeptanz von Gesetzmäßigkeiten“, bei dem Invarianzen als Begrenzungen des Handlungsspielraums verstanden werden, (2) Führung als „Nutzung von Gesetzmäßigkeiten“, bei dem Invarianzen als lediglich temporäre Begrenzungen des Handlungsspielraums verstanden werden und (3) Führung als „Verfügung über Gesetzmäßigkeiten“, bei dem Invarianzen als grundsätzlich durch Manipulation veränderbar verstanden werden. Aufbauend auf einer wissenschaftstheoretischen Analyse betriebswirtschaftlicher Aussagen plädieren wir dafür, Unternehmensführung als „Verfügung von Gesetzmäßigkeiten" zu verstehen, da die anderen beiden Auffassungen auf einem verkürzten Verständnis von Betriebswirtschaftslehre basieren.

Taking the debate on evidence-based management and success-factor research as a point of departure, this paper discusses three different views of the role of identified regularities for management practice: (1) acceptance of regularities, where regularities are understood as delimiting the scope of action, (2) use of regularities, where regularities are understood as restrictions that change over time and

(3) management of regularities, where regularities are understood as temporary invariances that can be managed by the firm. Reflecting on the scientific status of regularities in the social sciences, we argue that the first two views are based on a limited understanding of the nature of management sciences.

\section{Unternehmensführung als Einsicht in die Notwendigkeit?}

Mit unserem Titel nehmen wir Bezug auf den berühmten Hegelschen Ausspruch „Freiheit ist die Einsicht in die Notwendigkeit“. Zunächst wirkt der Satz falsch, denn: Notwendig- 
keiten schränken den Handlungsspielraum - und damit die Freiheit - ein. Beispielsweise reduziert die Einsicht in die (vermeintliche) Notwendigkeit bzw. Gesetzmäßigkeit, dass Unternehmen sich zum Überleben an ihre Umwelt anpassen müssen, den Handlungsspielraum der Unternehmensführung. So müssen z.B. die zu wählenden Strategien zur Umwelt passen, möchte das Unternehmen nicht ausselektiert werden. Um solche Notwendigkeiten der Unternehmensführung festzustellen, werden in der Betriebswirtschaftslehre zahlreiche quantitativ-empirische Studien durchgeführt. Diese untersuchen beispielsweise Erfolgsfaktoren von Unternehmensverkäufen (Defren/Wirtz 2010) und des Relationshipmarketings (Ryals/Knox 2001) und reichen von der Wirtschaftsinformatik (Wixom/Watson 2001) bis zur Strategieforschung (van Aaken et al. 2013). Hierbei werden in der Regel Beziehungen zwischen abhängigen und unabhängigen Variablen analysiert, um vor diesem Hintergrund entsprechende Gesetzmäßigkeiten aufzudecken. Im Idealfall wird die abhängige Variable durch die unabhängigen Variablen erklärt und somit eine vollständige Beschreibung der zugrunde liegenden Beziehungen erreicht. Diese werden dann als „Notwendigkeiten “ für die Führung von Unternehmen angesehen. In diesem Sinne zeichnet sich eine kluge Unternehmensführung durch eine Einsicht in die Notwendigkeit solcher betriebswirtschaftlicher Gesetzmäßigkeiten aus.

Der vorliegende Beitrag geht der Frage nach, ob man Unternehmensführung nur als eine solche „Einsicht in die Notwendigkeit“ verstehen sollte. Damit schließen wir an zwei sehr ähnlich geführten - Diskussionen an. Zum einen an die schon etwas ältere Debatte zur sog. „Erfolgsfaktorenforschung“ (Nicolai/Kieser 2002; Homburg/Krohmer 2004). Ausgangspunkt der Diskussion war das weit verbreitete Verständnis in der Betriebswirtschaftslehre, dass es in dieser darum gehe, Gesetzmäßigkeiten einer erfolgreichen Unternehmensführung aufzudecken (Bauer/Sauer 2004; Fritz 2004). Zum anderen hat sich vor kurzer Zeit eine ähnliche Debatte zum sog. „Evidence-Based Management“ entfacht, welche die Möglichkeit und Grenzen einer Übertragung der Methodologie evidenzbasierter Medizin in die Managementwissenschaften kritisch diskutiert (Rousseau 2006; Frank/ Kieser 2013; Frese 2013). Befürworter propagieren „strenge“ quantitativ-empirische Forschungsstandards zur Überbrückung der Kluft zwischen akademischer Rigorosität und praktischer Relevanz (Kuckertz 2012). Sie sehen in einem evidenz-basierten Management nicht nur die Standardmethodologie wissenschaftlicher Forschung, sondern auch einen vielversprechenden Ansatz zur Führung von Unternehmen (Pfeffer/Sutton 2006; Rousseau 2006).

Abgesehen von der Frage, ob die herrschende Forschungspraxis der evidenz-basierten Medizin überhaupt dem Ideal einer rigorosen Forschung entspricht (Ioannidis 2005; Young et al. 2008), argumentieren Kritiker der Übertragung dieser Standards auf die betriebswirtschaftliche Forschung, dass die Orientierung an der evidenz-basierten Vorgehensweise aus mindestens zwei Gründen problematisch ist. Zum einen werden die Standards der evidenz-basierten Medizin systematisch nicht erfüllt (womit ein Großteil der herrschenden Forschung als unwissenschaftlich gekennzeichnet werden müsste) und zum anderen können diese Standards gar nicht erfüllt werden (Learmonth 2006; Frank/Kieser 2013). Ein zentraler Grund dafür wird in der Komplexität der organisationalen Praxis gesehen, wodurch beobachtbare Wirkungen nicht ohne weiteres einer Managementintervention zugeschrieben werden können.

Anknüpfend an diese Diskussionen, wollen wir die Rolle von betriebswirtschaftlichen „Gesetzmäßigkeiten“ in der Unternehmensführung im Allgemeinen diskutieren. Wir wer- 
den zeigen, dass man es als Aufgabe der Unternehmensführung sehen kann, betriebswirtschaftliche Regelmäßigkeiten zu erkennen und aktiv darauf einzuwirken. Das heißt, es geht nicht nur um eine „Einsicht in die Notwendigkeit“ im Sinne einer Akzeptanz identifizierter Regelmäßigkeiten, sondern um die Reflexion der Regelmäßigkeiten und damit um die „Verfügung über (vermeintliche) Notwendigkeiten“.

Um dies zu erläutern, werden wir im nächsten Abschnitt zunächst kurz den erkenntnistheoretischen Status von wissenschaftlichen Sätzen in der Betriebswirtschaftslehre diskutieren. Wie in allen Sozialwissenschaften sind betriebswirtschaftliche Regelmäßigkeiten in ihre Geltung begrenzter als Gesetzmäßigkeiten naturwissenschaftlicher Disziplinen. Die Implikationen dieses Wissenschaftsverständnisses für die Führung von Unternehmen werden wir im darauf folgenden Abschnitt diskutieren. Unsere zentrale These lautet dort, dass die Unternehmensführung Ergebnisse quantitativ-empirischer Forschung berücksichtigen, diese aber nicht als unbedingte Notwendigkeiten interpretieren sollte. Eine Führung von Unternehmen besteht vielmehr in einem Balancieren zwischen der Akzeptanz von Notwendigkeiten und einem Brechen eben dieser. Schließen werden wir mit einem kurzen Ausblick, insbesondere auch auf die Lehre von der Unternehmensführung.

\section{Betriebswirtschaftliche Forschung als Suche nach Notwendigkeiten}

In der Diskussion um die wissenschaftstheoretische Basis der Betriebswirtschaftslehre ist es üblich, sich an den Naturwissenschaften zu orientieren und dementsprechend den Begriff der Theorie durch die in ihr enthaltenen Gesetzmäßigkeiten zu bestimmen (Stegmüller 1978; von Wright 1984; Zelewski 1999; Ruß 2004). Ein interessierendes Ereignis ist dann wissenschaftlich erklärt, wenn es deduktiv aus Gesetzmäßigkeiten hergeleitet werden kann (Hempel/Oppenheim 1948). Man kann auch sagen: Ein Ereignis wird erklärt, wenn es unter eine (oder mehrere) empirisch bestätigte allgemeine Gesetzesaussage(n) subsumiert werden kann. Sofern es gelingt, das Ereignis zu erklären, kann man auch Prognosen über zukünftige Ereignisse abgeben: Denn wenn man weiß, was zu einem bestimmten Ereignis geführt hat, weiß man auch um die Bedingungen, die eintreten müssen, damit das Ereignis in Zukunft wieder auftritt. In dieser Sicht besteht also zwischen Erklärung und Prognose kein logischer Unterschied. Zudem - und dies ist für die Betriebswirtschaftslehre als angewandte Wissenschaft besonders wichtig - geht man im Allgemeinen davon aus, dass man mithilfe dieses Wissens auch Gestaltungsempfehlungen generieren kann. Denn kann man bspw. den Erfolg eines Unternehmens durch dessen Investitionen in Innovationen erklären (vgl. z.B. Bae/Kim 2003), kann man nicht nur prognostizieren, dass andere Unternehmen, die ebenfalls in Innovationen investieren, erfolgreich werden, sondern man kann auch die unternehmerische Praxis gestalten, indem man darauf aufmerksam macht, dass wenn Unternehmen erfolgreich sein wollen, sie in Innovationen investieren sollten.

Im Gegensatz zu den Naturwissenschaften, bereitet es der Betriebswirtschaftslehre (wie auch anderen Sozialwissenschaften) erhebliche Probleme, dieser Auffassung von Theorie gerecht zu werden (vgl. Hollis 1994). Manche Wissenschaftler sehen darin ein Zeichen der Entwicklungsbedürftigkeit der Betriebswirtschaftslehre (Dannenbring/Starr 1981; Maurer 2004). Sie weisen darauf hin, dass die Tatsache, dass man bisher noch keine Gesetzmäßigkeiten in der Betriebswirtschaftslehre identifizieren konnte, nicht darauf schließen lasse, dass man nicht noch solche finden könne. Andere hingegen (Numagami 1998; Kirsch et al. 2007; Frank/Kieser 2013) argumentieren, dass es in der Betriebswirtschaftslehre keine gesetzesartigen Zusammenhänge geben könne und somit eine Ausrichtung an dem Ideal 
der naturwissenschaftlichen Forschung irreführend sei. Sie führen aus, dass Invarianzen immer nur unter bestimmten Voraussetzungen gelten; insbesondere gelten sie nur ceteris paribus, wenn also die Bedingungen, unter denen die Regelmäßigkeit postuliert wird, gleich bleiben. Sie entsprechen dem Grundsatz: „Wenn $\mathrm{x}$ - unter den Voraussetzungen $\mathrm{z}_{1}$, $\mathrm{z}_{2}, \mathrm{z}_{3}, \ldots, \mathrm{z}_{\mathrm{n}}$ - dann $\mathrm{y}$ “, wobei die Voraussetzungen $\mathrm{z}_{1}, \mathrm{z}_{2}, \mathrm{z}_{3}, \ldots, \mathrm{z}_{\mathrm{n}}$ (und dies ist ein zentraler Unterschied zu den Naturwissenschaften) Gegenstand evolutionärer Prozesse sind. Helmer und Rescher (1959) sprechen auch von „inexakten“ Wissenschaften, deren Wirkungszusammenhänge - im Vergleich zu exakten Wissenschaften - nicht als gültig im strengen Sinne interpretiert werden können, da unklar bleibt, ob und wie sie in konkreten Fällen anzuwenden sind. Sie folgern daraus $(1959,29)$ :

"An [inexact] law is thus not strictly universal in that it must be taken as applicable to all cases falling within the scope of its explicitly formulated conditions; rather it may be thought to formulate relationships which obtain generally, or better, as a rule."

Die Gründe, warum Regelmäßigkeiten in der Betriebswirtschaftslehre keine stabilen Kausalzusammenhänge darstellen, liegen in der Dynamik ihres Objektbereichs. Während man es in den Naturwissenschaften mit vergleichsweise konstanten „Phänomenen“ zu tun hat, zielt die Betriebswirtschaftslehre als Sozialwissenschaft darauf ab, menschliches Handeln zu erklären. Dieses ist durch so viele interdependente und veränderbare Faktoren bestimmt, dass es unmöglich erscheint, raumzeitlich invariante Muster ausfindig zu machen. So schreibt schon Hayek (1972, 34), dass Wirtschaftswissenschaftler mittlerweile eigentlich genug gelernt haben müssten, „um zu wissen, daß wir nicht all das wissen können, was wir zu einer vollständigen Erklärung der Phänomene wissen müßten.“ Dies bedeutet nicht, dass man aufgrund der dem menschlichen Handeln innewohnenden Dynamik und Komplexität keine wissenschaftlichen Erklärungen formulieren kann; es bedeutet aber schon, dass man den Anspruch an Erklärungen - im Vergleich zu exakten Wissenschaften - relativieren muss. Stellt man die verschiedenen Erklärungen gegenüber, ergibt sich folgendes Bild (vgl. Kirsch 1997, 250 ff.; Hübner 1986: 310 ff.):

\begin{tabular}{|l|l|}
\hline $\begin{array}{l}\text { Sozialwissenschaft, z.B. Betriebswirt- } \\
\text { schaftslehre }\end{array}$ & Naturwissenschaft, $\boldsymbol{z . B .}$ Physik \\
\hline $\begin{array}{l}\text { 1. Jemand war in einer bestimmten Lage. } \\
\begin{array}{l}\text { 2. Zu diesem Zeitpunkt glaubte er/sie an die } \\
\text { Geltung einer bestimmten Regel, nach der } \\
\text { man in solchen Lagen in bestimmter Weise } \\
\text { handeln müsse. }\end{array}\end{array}$ & $\begin{array}{l}\text { 1. Immer, wenn etwas in einer solchen Lage ist, } \\
\text { verändert es sich nach bestimmten Gesetzen. }\end{array}$ \\
\hline 3. Folglich handelte er/sie nach dieser Regel. & 3. Folglich ändert es sich nach diesen Gesetzen. \\
\hline
\end{tabular}

Tabelle 1: Erklärungen in den Sozial- und Naturwissenschaften

Das Wesentliche bei beiden Arten der Erklärung liegt in der zweiten Prämisse, die etwas völlig Unterschiedliches zum Ausdruck bringt: Bei der naturwissenschaftlichen Erklärung geht es um die Einführung einer „allgemeinen Gesetzeshypothese“, bei der betriebswirtschaftlichen Erklärung um eine Charakterisierung der individuellen „Definition der Situation“, die ein Individuum seinem/ihrem Handeln zugrunde legt. Durch die Abhängigkeit vom „Glauben“ der Individuen, unterliegen die Regelmäßigkeiten, welche die Betriebs- 
wirtschaftslehre formulieren kann, einer Kontingenz, die den exakten Wissenschaften fern ist. Damit weisen betriebswirtschaftliche Regelmäßigkeiten eine nur beschränkte Gültigkeit auf: Der Glauben in einer bestimmten Lage nach bestimmten Regeln handeln zu müssen (Prämisse 2), kann sich grundlegend ändern. Dabei müssen die Regeln, nach denen Individuen ihr Handeln ausrichten, den Individuen selbst nicht bewusst sein. Es spricht vieles dafür, dass ein Großteil menschlichen Verhaltens routinisiert ist. Insofern gelten beobachtete Regelmäßigkeiten solange, wie Individuen den Routinen tatsächlich folgen. Es wäre also kurzschlüssig, aus der relativierten Gültigkeit betriebswirtschaftlicher Zusammenhänge zu folgern, dass Ergebnisse "quantitativ-empirischer" Forschungen keine längerfristige Geltung beanspruchen dürfen (auch wenn in Routinen der Nukleus des Wandels bereits enthalten ist, vgl. Feldman/Pentland 2003). Wichtig ist die Erkenntnis, dass den in der Betriebswirtschaftslehre entdeckten Regelmäßigkeiten eine Kontingenz innewohnt, die eine Rede von starren „Gesetzmäßigkeiten“ obsolet werden lässt.

Ein zentraler Faktor, der die Kontingenz von Regelungen verursacht, sind sogenannte „selbst-zerstörende“ bzw. „selbst-erfüllende“ Prophezeiungen, deren wissenschaftliche Diskussion insbesondere John Venn (1888) zugeschrieben wird (Merton 1936). Dieser spricht von selbstzerstörende Prophezeiungen als ,annoying contingency to which prophets since the time of Jonah have been subject, (...); of publishing conclusions which are perfectly certain when every condition and cause but one have been taken into account, that one being the effect of the prophecy itself upon those to whom it refers" $(1888,226)$. Merton schließt draus, dass generell Planungen und Vorhersagen in den Sozialwissenschaften eine Kontingenz aufweisen, die den Naturwissenschaften fremd ist: Während Voraussagen in den Sozialwissenschaften den Objektbereich der Voraussage verändern und damit die Daten-(Basis) der Voraussage, ist dies in den Naturwissenschaften nicht der Fall. Merton (1936, 904) veranschaulicht den Unterschied anhand des Halleyschen Kometen:

"the prediction of the return of Halley's comet does not in any way influence the orbit of that comet; but (...) Marx's prediction of the progressive concentration of wealth and increasing misery of the masses did influence the very process predicted. For at least one of the consequences of socialist preaching in the nineteenth century was the spread of organizational labor, which, made conscious of its unfavorable bargaining position in cases of individual contract, organized to enjoy the advantages of collective bargaining, thus slowing up, if not eliminating, the developments which Marx had predicted."

Im Gegensatz zur den Naturwissenschaften reagiert der sozialwissenschaftliche Objektbereich auf Vorhersagen, weshalb Cueni und Frey (2014) auch von reaktiven Systemen sprechen. Galtung (1978, $100 \mathrm{ff}$.) veranschaulicht die Funktionsweise eines reaktiven Systems anhand der Milgram-Experimente (Milgram 1974), in welchen gezeigt wurde, dass Probanden auf Anweisung von Autoritätspersonen bereit sind, Handlungen auszuführen, welche den eigenen moralischen Vorstellungen widersprechen. Galtung argumentiert, dass die Versuchspersonen den Anweisungen nur so lange folgen, solange sie nicht wissen, dass sie Teil eines Experiments sind. In dem Moment, in dem man ihnen aber sagt, dass sie Teil eines Experiments sind, löst sich die beobachtete Regelmäßigkeit auf.

Aus Sicht des Ideals der naturwissenschaftlichen Forschung bestehen die „Kosten“ reaktiver Systeme darin, dass man bei der Erforschung dieser mit einem schwächeren Erklärungsbegriff auskommen muss (Küpper 1974; Kirsch 1997, 254). Mit anderen Worten: 
Das Erklärungs-, Prognose- und Gestaltungswissen, das die Betriebswirtschaftslehre generieren kann, entspricht nicht den strengen Vorstellungen einer exakten Naturwissenschaft. Erklärungen gelten nur ex post actu; sie sind für prognostische Zwecke nur dann ausreichend, wenn man davon ausgehen kann, dass alle Voraussetzungen, die bei der Erklärung einer Rolle spielten, in Zukunft nicht variiert werden. Diese Einschränkung gilt auch für das Gestaltungswissen. Transformiert man die Regelmäßigkeit „Wenn ein Unternehmen in Innovationen investiert, ist es erfolgreich“, in eine Gestaltungsempfehlung wie „Wenn das Unternehmen Erfolg haben möchte, muss in Innovationen investiert werden ", wird dies nur dann eintreffen, wenn alle Bedingungen unter denen diese Regelmäßigkeit „gefunden“ wurde, in Zukunft unverändert bleiben. Zu diesen Bedingungen zählt beispielsweise auch, dass nicht alle Unternehmen in Innovationen investieren, da dadurch die Quelle des Wettbewerbsvorteils - der Unterschied zum Wettbewerber - aufgehoben werden würde (Nicolai/Kieser 2002).

\section{Unternehmensführung als Reflexion von Notwendigkeiten}

Unsere bisherigen Ausführungen sollten zeigen, dass der Übertragung naturwissenschaftlicher Wissenschaftsstandards auf die Betriebswirtschaftslehre Grenzen gesetzt sind. In der Betriebswirtschaftslehre gibt es keine Gesetzmäßigkeiten, sondern „nur“ Regelmäßigkeiten. Anders als in den Naturwissenschaften kann also die betriebswirtschaftliche Forschung keine Naturkonstanten entdecken und damit Wissen schaffen, welche für alle Zeiten Gültigkeit beanspruchen könnte. Die Einsicht in die grundsätzliche Kontingenz von Regelmäßigkeiten hat auch Auswirkungen auf das Verständnis von Unternehmensführung, wie wir im Folgenden skizzieren wollen. Dazu unterscheiden wir drei unterschiedliche Verständnisse von Unternehmensführung, welche mit unterschiedlichen Reflexionsstufen im Umgang mit identifizierten Regelmäßigkeiten verbunden sind.

\subsection{Unternehmensführung als die Akzeptanz von Notwendigkeiten}

In einer ersten Stufe interpretiert die Unternehmensführung betriebswirtschaftliche Regelmäßigkeiten als Notwendigkeiten, die sie akzeptieren muss. So sprechen viele Studien von den „Gesetzmäßigkeiten des Marktes“ an welche sich Unternehmen anpassen müssten. Ein gutes Beispiel für eine solche Auffassung findet sich bei Deans et al. (2002), die auf der Basis einer großen quantitativen Studie zur Konsolidierung von Industrien Regelmäßigkeiten im Übernahmeverhalten von Unternehmen identifizieren. Sie argumentieren, dass diese Invarianzen Notwendigkeiten darstellen, an welche sich Unternehmen anpassen müssen, wenn sie überleben wollen. Sie schreiben:

„Nachdem wir die Gesetzmäßigkeiten der Industriekonsolidierung erkannt haben, können wir langfristige Voraussagen über Fusionen treffen, und noch konkreter können wir vorhersagen, welche Unternehmen als Übernahmekandidaten gelten. Wir können berechnen, welche Unternehmen in welchem Zeitraum konsolidiert werden. Aber dieses Muster zu erkennen, ist erst der Anfang. Die wichtigste Entdeckung ist die, dass Unternehmen mit der Endgames-Kurve in die Zukunft blicken - und in der Gegenwart die richtigen Entscheidungen treffen können. Damit können Unternehmen die Gesetzmäßigkeit der Endgames-Kurve nutzen, um selbst zu den Gewinnern zu gehören. " (Deans et al. 2002, 22f.) 
Versteht man Unternehmensführung wie Deans et al. als die Akzeptanz von Notwendigkeiten, ist es Aufgabe der Führung, den Gesetzen des Marktes zu folgen und dadurch zu den „Gewinnern“ zu gehören. Folgt man den Gesetzen nicht, so scheitert man zwangsläufig. Damit kann als einzig sinnvolle Bestrebung einer Führung nur eine Anpassung an gegebene Verhältnisse angesehen werden - ein Einfluss auf die Unternehmensentwicklung jenseits des reinen Nachvollziehens von Erfordernissen, die der Markt „verlangt“, ist nicht vorgesehen (vgl. auch Kirsch et al. 1979, 344 f.).

In einem solchen Verständnis von Unternehmensführung leistet die betriebswirtschaftliche Forschung einen wichtigen Beitrag zur Professionalisierung der Führung, da sie mittels quantitativ-empirischer Analysen die zu beachtenden Notwendigkeiten aufspürt. Etwas abgeschwächter, aber im selben Denken verhaftet, sind all diejenigen Ansätze, die das grundlegende Merkmal von Unternehmensführung darin sehen, Gesetzen des Marktes (oder denen einer erfolgreichen Unternehmensführung) zu folgen. Strategien, beispielsweise, werden dann als vorbestimmte Selektion von Handlungsmöglichkeiten interpretiert, die man angesichts der spezifischen Konfiguration von Unternehmen und Umwelt richtigerweise zu wählen hat. In diesem Sinne begreift die Unternehmensführung die zu verfolgende Strategie nicht als eine Wabl zwischen mehreren möglichen Strategien, sondern als das analytische Aufspüren der einen richtigen Strategie (vgl. auch Mintzberg et al. 2001; Dietel/Seidl 2003). Eine solch verstandene Unternehmensführung sieht ihre Aufgabe darin, durch eine Reflexion der herrschenden (Umwelt-) Bedingungen den Bereich der Handlungsmöglichkeiten auf die eine richtige Strategie zu beschränken.

\subsection{Unternehmensführung als das Nutzen von Notwendigkeiten}

Interpretiert man die Rolle der Unternehmensführung als ein „Nutzen von Notwendigkeiten“, versteht man betriebswirtschaftliche Zusammenhänge nicht als Gesetzmäßigkeiten, sondern lediglich als temporäre Regelmäßigkeiten. Insofern ist es zentrales Merkmal dieses Verständnisses, dass sich die Unternehmensführung der zeitlich begrenzten Gültigkeit empirischer Zusammenhänge bewusst ist: Regelmäßigkeiten werden nicht als gegeben hingenommen, sondern als Wirkungszusammenhänge interpretiert, die Veränderungen unterliegen. Damit werden der Unternehmensführung wesentlich mehr Freiheitsgrade zugesprochen. Die Rolle der Unternehmensführung beschränkt sich nicht auf eine „blinde“ Akzeptanz von Notwendigkeiten; eine Führung von Unternehmen besteht vielmehr darin, Regelmäßigkeiten ausfindig zu machen und zu nutzen, ohne sich zu sehr auf die Geltung bestimmter Zusammenhänge zu verlassen (vgl. auch Gross 1999). Ist es z.B. strategisches Ziel eines Unternehmens, zu den drei größten Wettbewerbern auf jedem Markt zu zählen, in dem es tätig ist, wird es als ein Zeichen „kluger“ Unternehmensführung gewertet werden, wenn diese die festgestellten positiven empirischen Zusammenhänge zwischen Markteintrittszeitpunkt und Marktanteil nutzt (vgl. z.B. Szymanski et al. 1995). Dabei wird die Unternehmensführung sich aber nicht auf die Gültigkeit der angenommen Zusammenhänge verlassen, sondern diese immer wieder überprüfen. So könnte es beispielsweise sein, dass bestimmte technische Voraussetzungen, unter denen die Erfolgswirksamkeit des Markteintrittszeitpunktes festgestellt worden ist, nicht mehr gelten.

Eines der prominentesten Beispiele für ein Verständnis von Unternehmensführung als ein Nutzen von Notwendigkeiten ist Porters „market-based view“ (vgl. Porter 1985). Auf der einen Seite macht Porter deutlich, dass sich die Positionierung des Unternehmens an gegenwärtig geltende Branchengegebenheiten auszurichten hat (und somit die Akzeptanz 
von Notwendigkeiten eine große Rolle spielt). Auf der anderen Seite sind brancheninterne Machtverhältnisse nicht stabil gegeben. Da die Wettbewerbskräfte dynamisch wirken und so z.B. immer wieder neue Konkurrenten in einen Markt drängen, neue Technologien die Gefahr einer Substitution erhöhen usw., sind Unternehmen gut beraten, die Entwicklung von Wettbewerbskräften in ihrem Umfeld zu beobachten und gegebenenfalls ihr Unternehmen neu auszurichten. Bei aller Umweltdynamik bleibt aber klar, dass Unternehmensführung sich an bestimmte Regeln zu halten hat: Unternehmen müssen in der einen oder anderen Weise anhand von „generischen“ Strategien eine kluge und konsistente Positionierung innerhalb der Branche einnehmen, da sie sich sonst in „Widersprüchen“ verstricken (Porter 1996, 67) und aus dem Wettbewerb ausscheiden. Die Freiheitsgrade der Positionierung sind demnach beschränkt.

Das hier skizzierte Verständnis von Unternehmensführung als ein „Nutzen von Notwendigkeiten“ setzt gegenüber dem Verständnis einer „Akzeptanz von Notwendigkeit“ ein erhöhtes Reflexionsniveau der Unternehmensführung voraus. Denn Führung sollte nicht nur die für das Unternehmen wichtigen empirischen Zusammenhänge in Erfahrung bringen und im Unternehmen durchsetzen, sondern auch zusätzlich die Gültigkeit dieser Zusammenhänge kritisch hinterfragen. Damit geht mit dem gesteigerten Reflexionsniveau auch die Gefahr eines „Scheiterns“ der Unternehmensführung einher, da sich letztendlich beide Kontrollerfordernisse konterkarieren können: Auf der einen Seite muss die Führung auf eine konsequente Umsetzung ihrer Vorhaben achten, auf der anderen Seite muss sie immer wieder die Prämissen ihrer Vorhaben prüfen. Insofern ist sie mit einem „Dilemma der Kontrolle" konfrontiert (Kirsch et al. 2009, 218; Schreyögg/Steinmann 1987), da die Infragestellung von Prämissen in der Regel einer konsequenten Umsetzung genauso entgegenläuft, wie eine konsequente Umsetzung der Infragestellung von Prämissen.

\subsection{Unternehmensführung als das Verfügen über Notwendigkeiten}

Die dritte Auffassung knüpft an dem Verständnis des „Nutzens von Notwendigkeiten“ an, erweitert dieses aber noch in einer zentralen Hinsicht: Die Geltung betriebswirtschaftlicher Regelmäßigkeiten ist nicht nur zeitlich begrenzt; Regelmäßigkeiten liefern immer auch Hinweise, wie sie vom Management durchbrochen werden können (Galtung 1978; Kirsch et al. 2008). Insofern betont das Verständnis von Unternehmensführung als das „Verfügen über Regelmäßigkeiten“ die mit einer Regelmäßigkeit einhergehende Kontingenz. Im letzten Abschnitt haben wir argumentiert, dass Regelmäßigkeiten in der Betriebswirtschaftslehre auf Voraussetzungen beruhen, die selbst nicht invariant sind. In diesem Sinne geht es bei der Führung von Unternehmen nicht nur darum, zu reflektieren, auf welchen Voraussetzungen die bestehenden Regelmäßigkeiten beruhen und ob diese sich verändert haben, sondern auch unter welchen Umständen diese Voraussetzungen „gebrochen“ werden könnten. Damit sind Regelmäßigkeiten nicht als ein Datum zu interpretieren, mit dem das Management umzugehen hat, sondern als ein kontingentes Handlungsmuster, welches grundsätzlich wählbar erscheint.

Es sind einige Fälle bekannt, in denen insbesondere kleinere Unternehmen ganze Branchen neu strukturiert und damit den Wettbewerb neu geordnet haben (vgl. bspw. Napster, das die Musikbranche revolutionierte). Auslöser dieser „schöpferischen Zerstörungen“ (Schumpeter 1950) sind oft sog. „disruptive Technologien“ (Christensen/Bower 1996), welche so bahnbrechende Innovationen darstellen, dass sie als notwendig angenommene Regelmäßigkeiten durchbrechen. Konventionelle Branchenanalysen werden bei Analyse 
dieser Unternehmen zu falschen Ergebnissen kommen, weil sie auf gegenwärtig bekannten Kundenbedürfnissen beruhen und keine zukünftigen Kundenbedürfnisse berücksichtigen (können). Dies zeigt auch der „Honda-Fall“, der es im strategischen Management zu einiger Berühmtheit geschafft hat (Mintzberg et al. 2001; 139-140). Ende der 1970'er Jahre stand Honda vor der Entscheidung in das Autogeschäft einzusteigen. Gemäß klassischer Branchenanalyse war der Automarkt unattraktiv, denn der Markt war zum damaligen Zeitpunkt gesättigt, es gab starke Konkurrenten und Honda hatte keine Kompetenzen im Automobilgeschäft. Aber obwohl diese Interpretation aus Sicht des „Market-based View“ richtig war, ist Honda dennoch sehr erfolgreich ins Automobilgeschäft eingestiegen.

Während das Verständnis von Unternehmensführung als „Akzeptanz von Notwendigkeiten“ genauso wie das der „Nutzung von Notwendigkeiten“ stark von der Vorstellung gegebener Notwendigkeiten ausgeht, verlangt das Verständnis eines „Verfügens über Notwendigkeiten", dass man solche Notwendigkeiten als vermeintliche hinterfragt. Dies gilt ausnahmslos für sämtliche Notwendigkeiten, die bei einer Führung von Unternehmen als selbstverständlich angenommen werden. Unternehmensführung heisst dann ein Verfügen über Notwendigkeiten. Schnädelbach beschreibt dieses Verfügen als Ausdruck rationales Handelns, wenn er schreibt:

„Intuitiv ist klar, daß nur derjenige rational handelt, der nicht blo $\beta$ Regeln folgt, sondern auch noch über diese Regeln selbst und das Verfügen über sie verfügt, und auch über dieses Verfügen verfügt usf. ins Unendliche; jede Grenze des Verfügens über das Regelverfügen wäre eine Rationalitätsschranke." (Schnädelbach 1987, 81)

In diesem Sinne ist eine Unternehmensführung dazu angehalten, sich vermeintlichen Notwendigkeiten nicht vorschnell zu beugen, sondern die Voraussetzungen dieser zu reflektieren. Je ausgeprägter der Reflexionsgrad, desto deutlicher wird die Kontingenz der Notwendigkeiten bzw. desto deutlicher erscheinen diese Notwendigkeiten selbst als wählbar und damit im Schnädelbach“schen Sinne „verfügbar“. Bei voll ausgeprägtem Reflexionsgrad werden die zugrunde liegenden Notwendigkeiten selbst als Selektion verstanden.

Im Umkehrschluss impliziert dieses Verständnis von Unternehmensführung aber nicht, dass man z.B. marktwirtschaftliche Prinzipien ignorieren bzw. frei über diese verfügen kann. Im Gegensatz zu anderen Konzeptualisierungen von Unternehmensführung wird allerdings betont, dass die Führung von Unternehmen keinen Gesetzmäßigkeiten „ausgeliefert“ ist, sondern grundsätzlich die Entwicklung des Unternehmens selbst vorantreiben kann. Anders formuliert: Je weniger reflektiert die Unternehmensführung ist, desto mehr unterliegt sie der Illusion, es gäbe Notwendigkeiten, über die man nicht selber entscheiden könne. Zudem geht mit dem Reflexionsgrad eine zunehmende Flexibilität einher (vgl. auch Dietel/Seidl 2003). Denn je reflektierter die Führung von Unternehmen erfolgt, desto deutlicher wird die Kontingenz ihrer Entscheidungen, d.h. die Möglichkeit, auch andere Entscheidungen treffen zu können.

\section{Ausblick: Unternehmensführung lehren?}

Das Verständnis von Unternehmensführung variiert mit dem Glauben an die Existenz und Gültigkeit betriebswirtschaftlicher Invarianzen. Wir plädieren dafür, Betriebswirtschaftslehre als Sozialwissenschaft zu begreifen und damit Unternehmensführung als ein „Verfügen von Regelmäßigkeiten“ zu verstehen. Dies bedeutet nicht, dass quantitativ-empirische Forschung überflüssig wäre. Im Gegenteil, alle hier ausgeführten Verständnisse bauen auf 
der Suche und Bestätigung von Regelmäßigkeiten auf. Insofern stellt das Aufspüren von empirischen Wirkungszusammenhängen eine zentrale Aufgabe betriebswirtschaftlicher Forschung dar.

Auch eine professionelle Unternehmensführung in der Praxis zeichnet sich unseres Erachtens durch ein Erkennen und Nutzen von Regelmäßigkeiten aus. So basieren alle gängigen Managementsysteme wie Planungs- und Kontrollsysteme oder Vergütungs- und Informationssysteme mit den jeweils dahinterstehenden Modellen auf der Annahme, dass Organisationen und deren Umwelten in kausalen Zusammenhängen miteinander agieren. Unternehmen können daher ohne die Annahme, dass bestimmte Regelmäßigkeiten gelten, nicht professionell geführt werden. Das in diesem Beitrag skizzierte Verständnis von Unternehmensführung geht nun jedoch noch einen Schritt weiter, indem wir nicht nur das Nutzen, sondern auch das Verfügen über Regelmäßigkeiten als Charakteristikum einer professionalisierten Unternehmensführung ansehen. Unternehmensführung ist dann nicht nur durch die Verwendung einschlägiger Managementsysteme gekennzeichnet, sondern gerade auch durch die Reflexion der Möglichkeiten und Grenzen solcher Managementsysteme. So werden „schöpferische Zerstörungen“, „disruptive Technologien“ oder „radikale Innovationen“ nur erfolgreich von einem Unternehmen entdeckt bzw. umgesetzt werden können, wenn Manager die bisher als invariant angenommen Zusammenhänge durchbrechen. Napster, amazon.com und Apple sind prominente Beispiel für Unternehmen, die dies geschafft haben.

Wir glauben, dass es gerade Aufgabe der universitären Lehre ist, die Voraussetzungen, auf denen die betriebswirtschaftlichen Instrumente und Theorien beruhen, den Studierenden - und damit den späteren Managern - immer wieder klar vor Augen zu führen. Nur so kann der Grundstein für eine Reflexion dieser und eine Verfügung über sie gelegt werden. Die Reflexion von Notwendigkeiten erfolgt nicht um ihrer selbst willen, sondern in der Absicht, neue und eventuell „bessere“ Handlungsoptionen konstruieren zu können. Eine Lehre der Unternehmensführung umfasst dann sowohl die Lehre der Instrumente aus dem „Werkzeugkasten“ der Betriebswirtschaftslehre als auch die Schulung einer Kompetenz der Infragestellung der eigenen Denkvoraussetzungen. Zentral muss dabei die Einsicht sein, dass die Geltung gegenwärtig „messbarer“ Regelmäßigkeiten sowie gegenwärtig anerkannter Theoriezusammenhänge begrenzt ist. Radikale Neuerungen in der Unternehmens- als auch in der Wissenschaftspraxis kann man nicht aus vergangenen Regelmäßigkeiten ableiten.

\section{Literaturverzeichnis}

Aaken, D. van, et al. (2013): Ausgestaltung und Erfolg von Stratgieworkshops: eine empirische Analyse, in: Zeitschrift für betriebswirtschaftliche Forschung, Jg. 65, S. 588-616.

Bae, S./Kim, D. (2003): The effects on R\&D Investments on Market Value of firms: Evidence from the U.S., Germany, and Japan. The Multinational Business Review, Jg. 11, S. 51-75.

Bauer, H.H./Sauer, N.E. (2004): Die Erfolgsfaktorenforschung als schwarzes Loch?, in: Die Betriebswirtschaft, Jg. 64, S. 621-623.

Christensen, C.M./Bower, J.L. (1996): Customer Power, Strategic Investment, and the Failure of Leading Firms, in: Strategic Management Journal, Jg. 17, Nr. 3, S. 197-218.

Cueni, R./Frey, B.S. (2014): Forecasts and Reactivity, in: Crema Working Paper No. 2014-10.

Dannenbring, D./Starr, M. (1981): Management Science: An Introduction. New York. 
Deans, G., et al. (2002): Merger Endgames - Strategien für die Konsolidierungswelle. Wiesbaden.

Defren, T./Wirtz, B.W. (2010): Erfolgsfaktoren beim Verkauf von Unternehmensteile, in: Die Unternehmung, Jg. 64, S. 239-276.

Dietel, B./Seidl, D. (2003): Überlegungen zu einem allgemeinen Strategiebegriff, in: Ringlstetter, M., et al. (Hrsg. 2003): Perspektiven des Strategischen Managements - Theorien, Konzepte, Anwendungen. Wiesbaden, S. 25-42.

Feldman, M. S./Pentland, B.T. (2003): Reconceptualizing Organizational Routines as a Source of Flexibility and Change, in: Administrative Science Quarterly, Jg. 48, S. 94-118.

Frank, G./Kieser, A. (2013): Kann man Managementwissenschaft nach dem Muster der Evidenzbasierten Medizin betreiben?, in: Die Betriebswirtschaft, Jg. 73, S. 167-181.

Frese, M. (2013): Es gibt so viel zu tun, warum gegen Evidenzbasiertes Management polemisieren?, in: Die Betriebswirtschaft, Jg. 73, S. 221-231.

Fritz, W. (2004): Die Erfolgsfaktorenforschung - ein Misserfolg?, in: Die Betriebswirtschaft, Jg. 64, S. 623-625.

Galtung, J. (1978): Methodologie und Ideologie. Frankfurt a. M.

Gross, P. (1999): Management in der Multioptionsgesellschaft, in: Gomez, P, et al. (Hrsg. 1999): Entwicklungsperspektiven einer integrierten Managementlehre. Bern, S. 37-56.

Hayek, F.A. (1972): Die Theorie komplexer Phänomene. Tübingen.

Helmer, O./Rescher, N. (1959): On the Epistemology of the Inexact Sciences. Management Science, Jg. 6, S. 25-52.

Hempel, C.G./Oppenheim, P. (1948): Studies in the Logic of Explanation, in: Philosophy of Science, Jg. 15, S. 135-175.

Hollis, M. (1994): The Philosophy of Social Science. Cambridge.

Homburg, C./Krohmer, H. (2004): Die Fliegenpatsche als Instrument des wissenschaftlichen Dialogs, in: Die Betriebswirtschaft, Jg. 64, S. 626-631.

Hübner, K. (1986): Wissenschaftliche Vernunft und Post-Moderne, in: Koslowski, P., et al. (Hrsg. 1986): Moderne oder Postmoderne? Zur Signatur des gegenwärtigen Zeitalters. Weinheim, S. 63-78.

Ioannidis, J. P.A. (2005): Why Most Published Research Findings Are False, in: PLoS Medicine 2(8): e124.

Kirsch, W. (1997): Kommunikatives Handeln, Autopoiese, Rationalität. Herrsching.

Kirsch, W., et al. (1979): Das Management des geplanten Wandels. Stuttgart.

Kirsch, W., et al. (2007): Betriebswirtschaftliche Forschung. Stuttgart.

Kirsch, W., et al. (2008): Werte und Innovationen - Überlegungen vor dem Hintergrund einer trilateralen Wissenschaftskonzeption, in: Sackmann, S. (Hrsg. 2008): Mensch und Ökonomie, Wiesbaden, S. 190-205.

Kirsch, W., et al. (2009): Unternehmensführung. Eine evolutionäre Perspektive. Stuttgart.

Küpper, H.-U. (1974): Grundlagen einer Theorie der betrieblichen Mitbestimmung. Berlin.

Kuckertz, A. (2012): Evidence-based Management - Mittel zur Überbrückung der Kluft von akademischer Strenge und praktischer Relevanz?, in: Zeitschrift für betriebswirtschaftliche Forschung, Jg. 64, S. 803-827.

Learmonth, M. (2006): Is There Such a Thing As Evidence-Based Management?: A Commentary on Rousseau's 2005 Presidential Address, in: Academy of Management Review, Jg. 31, S. 1089-1091. 
Maurer, R.W. (2004): Zwischen Erkenntnisinteresse und Handlungsbedarf - Eine Einführung in die methodologischen Probleme der Wirtschaftswissenschaft. Marburg.

Milgram, S. (1974): Obedience to Authority. An Experimental View. New York.

Mintzberg, H., et al. (2001): Strategy Safari. Wien.

Nicolai, A./Kieser, A. (2002): Trotz eklatanter Erfolgslosigkeit: die Erfolgsfaktorenforschung weiter auf Erfolgskurs, in: Die Betriebswirtschaft, Jg. 62, S. 579-596.

Numagami, T. (1989): The Infeasibility of Invariant Laws in Management Studies: A Reflective Dialogue in Defense of Case Studies, in: Organization Science, Jg. 9 (1), S. 2-15.

Porter, M. (1985): Competitive Advantage: Creating and Sustaining Superior Performance. New York.

Porter, M. (1996): What is Strategy?, in: Harvard Business Review, Jg. 74, Nr. 6, S. 61-78.

Pfeffer, J./Sutton, R. (2006): Evidence-Based Management, in: Harvard Business Review, Jg. 84, Nr. 1, S. 62-74.

Rousseau, D.M. (2006). Is there such a thing as "Evidence-based Management" ?, in: Academy of Management Review, Jg. 31, S. 256-269.

Ruß, H.-G. (2004): Wissenschaftstheorie, Erkenntnistheorie und die Suche nach der Wahrheit. Stuttgart.

Ryals, L./Knox, S. (2001): Cross-functional issues in the implementation of relationship marketing through customer relationship management, in: European Management Journal, Jg. 19, S. 534-542.

Schnädelbach, H. (1987): Über Rationalität und Begründung. Philosophie und Begründung. F. f. P. B. Homburg. Frankfurt a. M., S. 67-83.

Schreyögg, G./Steinmann, H. (1987): Strategic Control: A new Perspective, in: Academy of Management Review, Jg. 12, Nr. 1, S. 91-103.

Schumpeter, J.A. (1950): Capitalism, Socialism and Democracy. New York.

Stegmüller, W. (1978): Hauptströmungen der Gegenwartsphilosophie. Stuttgart.

Szymanski, D., et al. (1995): Order of Entry and Business Performance: An Empirical Synthesis and Reexamination, in: Journal of Marketing, Jg. 59, Nr. 4, S. 17-33.

Venn, J. (1888): The Logic of Chance. An Essay on the Foundations and Province of the Theory of Probability with especial Reference to its Logical Bearings and Applications to Moral and Social Science, and to Statistics. London.

von Wright, G.H. (1984): Erklären und Verstehen, Frankfurt a. M.

Wixom, B./Watson, H. (2001): An empirical investigation of the factors affecting data warehousing success, in: MIS Quarterly, Jg. 25, Nr. 1, S. 17-32.

Young, N., et al. (2008): Why Current Publication Practices may Distort Science, in: PLoS Medicine 5, e201.

Zelewski, S. (1999): Grundlagen, in: Corsten, H./Reiß., M. (Hrsg. 1999): Betriebswirtschaftslehre, München, S. 1-125. 
Dominik van Aaken, Dr. habil. ist wissenschaftlicher Oberassistent an der Munich School of Management, Universität München.

Anschrift: Universität München, Ludwigstr. 28, 80539 München, Deutschland, Tel.: + 49 892180 2879, email: aaken@bwl.lmu.de

Werner Kirsch, Dr. Dres. h.c., ist emeritierter Professor für Unternehmenspolitik und strategische Führung und Leiter des Zentrums für organisationstheoretische Grundlagenforschung an der Munich School of Management, Universität München.

Anschrift: Universität München, Zentrum für organisationstheoretische Grundlagenforschung, Ludwigstr. 28, 80539 München, Deutschland

David Seidl, PhD., ist Professor für Organisation und Management an der Universität Zürich.

Anschrift: Universität Zürich, Lehrstuhl für Organisation und Management, Universitätsstrasse 84, 8006 Zürich, Schweiz, Tel: +41 4463437 52, email: david.seidl@business.uzh.ch 